\title{
Mark Spivak (1929-1998). In memoriam
}

\author{
LORENZO BURTI
}

Il 5 novembre scorso Mark Spivak è morto a Gerusalemme all'età di 69 anni, combattendo l'ultima battaglia contro la malattia e le disabilità fisiche che lo hanno accompagnato per gran parte della sua vita.

Egli è ben noto nel nostro paese per i suoi contributi importanti alla teoria e pratica della riabilitazione psicosociale, esposti nell'arco degli ultimi quattro lustri con la chiarezza, la pazienza, la meticolosità e la profonda umanità che chi ha partecipato ai suoi seminari non potrà mai dimenticare.

Mark Spivak era nato a Chicago nel 1929 da famiglia ebraica, si era laureato in psicologia alla Columbia University nel 1955 ed aveva conseguito il dottorato in psicologia sociale all'Università del Michigan nel 1962. Si era trasferito in Israele nel 1966 dove, dal 1968 al 1980 dirigeva un centro di riabilitazione psicosociale per pazienti psichiatrici gravi diventato famoso, il Moadon Shalom di Gerusalemme. Dal 1983 al 1985 dirigeva un programma riabilitativo dei Servizi di Psicologia dell'Università Ebraica di Gerusalemme. Successivamente, nonostante l'aggravarsi delle condizioni fisiche, conduceva un'intensa attività didattica e di consulenza sia in patria che all'estero, attività che lo ha portato numerose volte, fino agli ultimi tempi, nel nostro paese.

Nel periodo del dottorato, dal 1956 al 1961, aveva lavorato come psicologo sociale ricercatore presso l'ospedale psichiatrico statale di Ypsilanti, nel Michigan, sviluppando quell'interesse clinico e scientifico per i cosiddetti pazienti psichiatrici cronici gravi che avrebbe improntato tutta la sua carriera professionale, e acquisendo quell'esperienza pregnante che solo il manicomio conferisce a chi vi lavora in modo critico e innovativo. Nelle prime pubblicazioni, dal 1960 al 1967, iniziava a delineare quel modello di riabilitazione psicosociale che avrebbe poi sperimentato e affinato al Moadon Shalom. Il Moadon, un centro di riabilitazione nato con fondi di ricerca del NIMH e legato quindi ai destini della ricerca stessa (per que- sto venne chiuso nel 1980 , quando era al massimo del successo), diventava il punto di riferimento per la riabilitazione dei pazienti gravi, in un contesto di servizi peraltro molto tradizionale, ospedalocentrico e manicomiale.

Nel modello riabilitativo elaborato da Spivak la cronicità non è semplicemente la conseguenza di un deficit dell'individuo, da correggere con interventi educativi, ma si sviluppa, e si mantiene nell'interazione reciproca fra la mancanza di competenze sociali dell'individuo e la disfunzionalità delle interazioni tra l'individuo e le persone attorno a lui. Quindi non cronicità, ma processi cronici; non esito difettuale, ma equilibrio attivamente mantenuto a livelli bassi di articolazione sociale dai medesimi processi che iniziarono la disfunzione. «Perciò - sostiene Spivak -, il compito della prevenzione, della terapia e della riabilitazione è uno solo e sempre quello: neutralizzare $\mathbf{i}$ processi cronici che, partendo dal fallimento nei rapporti sociali, portano all'isolamento, alla desocializzazione e alla rassegnazione». La dimensione interpersonale attraversa tutto il modello riabilitativo di Spivak, che trova nel rapporto terapeutico la sua vera essenza. In ciò si discosta da altri modelli riabilitativi di stampo cognitivo-comportamentale, che sottolineano gli aspetti didattici talora a scapito di quelli umani, rischiando di diventare riduttivi e meccanicistici.

L'umanità è forse il carattere saliente della riabilitazione di Mark Spivak, come del resto lo era dell'Uomo Mark. Forse anche perché egli viveva quotidianamente nel proprio fisico il dolore e il dramma della disabilità. Sin da bambino, infatti, a causa di un incidente, intraprendeva un calvario di interventi chirurgici a catena, che comunque lasciavano come esito un grave difetto della deambulazione. La natura continuava ad accanirsi con il suo fisico anche da adulto, quando sopraggiungevano una malattia cronica invalidante e una serie di problemi, soprattutto alla vista, che trasformavano in un impegno sovru- 
mano qualsiasi attività non sedentaria, figuriamoci i suoi spostamenti per il mondo ad insegnare! Mark era un esempio vivente di lotta alla disabilità e alla rinuncia, prima ancora che esperto di riabilitazione!

Nel 1980 venne invitato nel Veneto per una serie di seminari rimasti memorabili, e fu un amore a prima vista per l'Italia. Mark ha insegnato in moltissimi paesi, dagli Stati Uniti all'Estremo Oriente, ma l'Italia era il suo luogo ideale. Gli piaceva tutto, dal carattere della gente, all'arte, alla cucina. Nel 1987 e nel 1997 riusciva a trascorrere alcuni mesi sabbatici a Verona, città che amava tantissimo, tanto da farsi chiamare scherzosamente «Marcello da Verona»; arrivava al punto di firmare così gli articoli pubblicati nel nostro paese.

La sua serietà e tenacia a livello professionale stupiva anche il meno interessato ed attento ai problemi della riabilitazione, e la sua motivazione e spinta trascinavano anche i più pigri. Prima di un seminario in un luogo nuovo si presentava al servizio di buon mattino per vedere tutto di persona, per poter cosi impostare la successiva trattazione e discussione in modo massimamente rilevante per i bisogni specifici di quel servizio, di quella realtà. Non riusciva a capacitarsi, e non accettava, di venire invitato ad un congresso, ad un'inaugurazione per semplice rappresentanza, per essere esibito all'assessore in carica. Si lamentava che, nello stile grandioso e celebrativo che spesso ci caratterizza, venisse accordato troppo tempo ai momenti ufficiali e conviviali, rispetto a quello riservato alla effettiva formazione degli operatori e, caparbio qual era, non mancava di stupire o irritare l'anfitrione di turno pretendendo di parlare in orari impossibili con gli operatori del servizio. Ricordiamo cosi Mark, con affetto, riconoscenza, e un profondo senso di perdita. Ci rimangono i suoi insegnamenti attraverso i seminari, i suoi articoli, alcuni dei quali, fondamentali, anche in italiano. E soprattutto il suo esempio, che ci incoraggia a non cedere mai alla malattia e alle avversità della vita. 